\title{
Temperature and cooling age of the white dwarf companion of PSR J0218+4232
}

\author{
C. G. Bassa ${ }^{1}$, M. H. van Kerkwijk ${ }^{1, \star}$, and S. R. Kulkarni ${ }^{2}$ \\ 1 Astronomical Institute, Utrecht University, PO Box 80000, 3508 TA Utrecht, The Netherlands \\ 2 Palomar Observatory, California Institute of Technology 105-24, Pasadena, CA 91125, USA
}

Received 7 February 2003 / Accepted 6 March 2003

\begin{abstract}
We report on Keck optical BVRI images and spectroscopy of the companion of the binary millisecond pulsar PSR J0218+4232. A faint bluish $(V=24.2, B-V=0.25)$ counterpart is observed at the pulsar location. Spectra of this counterpart reveal Balmer lines which confirm that the companion is a helium-core white dwarf. We find that the white dwarf has a temperature of $T_{\text {eff }}=8060 \pm 150 \mathrm{~K}$. Unfortunately, the spectra are of insufficient quality to put a strong constraint on the surface gravity, although the best fit is for low $\log g$ and hence low mass $\left(\sim 0.2 M_{\odot}\right)$, as expected. We compare predicted white dwarf cooling ages with the characteristic age of the pulsar and find similar values for white dwarf masses of 0.19 to $0.3 M_{\odot}$. These masses would imply a distance of 2.5 to $4 \mathrm{kpc}$ to the system. The spectroscopic observations also enable us to estimate the mass ratio between the white dwarf and the pulsar. We find $q=7.5 \pm 2.4$, which is consistent with the current knowledge of white dwarf companions to millisecond pulsars.
\end{abstract}

Key words. pulsars: individual: PSR J0218+4232 - stars: neutron - stars: white dwarfs

\section{Introduction}

Millisecond or recycled pulsars are almost always found in binaries. Their short (millisecond) periods are believed to be caused by the accretion of matter from the binary companion, which spins up the neutron star, and, by means poorly understood, decreases its magnetic field. This accretion will happen when the companion, in the course of its evolution, overfills its Roche lobe.

When the companion of the pulsar is a white dwarf, one would expect the cooling age of the white dwarf to match the age of the pulsar. This because the cooling and spin-down clocks start ticking at roughly the same time, when the companion starts to contract to a white dwarf and the pulsar turns on following the cessation of mass transfer.

The age of a spin-down powered pulsar, with period $P$ and period derivative $\dot{P}$, is given by

$$
\tau_{\mathrm{PSR}}=\frac{P}{(n-1) \dot{P}}\left[1-\left(\frac{P_{0}}{P}\right)^{n-1}\right],
$$

where $P_{0}$ is the initial period and $n$ is the braking index. The latter determines how the rotational energy loss scales with the period $\left(\dot{P} \propto P^{2-n}\right)$. For magnetic dipole radiation (see

Send offprint requests to: C. G. Bassa,

e-mail: c.g.bassa@astro.uu.nl

* Present address: Dept. of Astronomy and Astrophysics, Univ. of Toronto, 60 St George Street, Toronto, ON M5S 3H8, Canada; e-mail: mhvk@astro.utoronto.ca
Lyne \& Smith 1998) we have $n=3$, and if we further assume that $P_{0} \ll P$, the pulsar spin-down age is given by $\tau_{\text {char }}=P /(2 \dot{P})$, the "characteristic age" of the pulsar. The uncertainty in the pulsar age is caused by $n$ and $P_{0}$, where, for the former, values lower than 3 will lead to ages longer than the characteristic age, while initial periods close to the current period will lead to shorter ages.

Another possible source of uncertainty in the characteristic pulsar age is the transverse velocity of the pulsar, which causes an apparent acceleration that contributes to the observed period derivative. Ignoring this 'Shklovskii' effect (Shklovskii 1970) can lead to significant underestimates of the characteristic age (Camilo et al. 1994).

The cooling age of the white dwarf can be determined from its effective temperature and its mass, using a cooling model. With the optical detection of several white dwarf companions to millisecond pulsars (e.g., Danziger et al. 1993; Nicastro et al. 1995; Lundgren et al. 1996a,b), considerable effort has been spent in constructing such cooling models (Hansen \& Phinney 1998a; Driebe et al. 1998; Serenelli et al. 2001). It was found that the main uncertainty in predicting white dwarf cooling ages is the thickness of the hydrogen envelope surrounding the helium-core. Thick envelopes can support residual hydrogen shell burning, which slows the cooling of the white dwarf, while thin layers cannot and thus lead to faster cooling.

Hydrogen shell burning can become unstable, leading to thermonuclear flashes, which may reduce the mass of the 
envelope until it cannot sustain hydrogen fusion any longer. It was already found by Webbink (1975) that these thermal flashes do not occur for white dwarfs with masses below $\sim 0.2 M_{\odot}$, a result that subsequently has been confirmed by Alberts et al. (1996), Driebe et al. (1998), Sarna et al. (2000), and Althaus et al. (2001). This limit is due to the fact that flashes are caused by unstable hydrogen burning through the CNO cycle (Driebe et al. 1999) which does not occur for stars with masses below $\sim 0.2 M_{\odot}$.

Above this limit all authors do find flashes, but the consequences differ. Driebe et al. (1998) find that the flashes do not reduce the envelope mass sufficiently to suppress residual hydrogen shell burning and hence they predict long cooling ages for masses also above $0.2 M_{\odot}$. In a later paper, however, Schönberger et al. (2000) suggest this result might be invalid for closer binaries, in which the mass of the envelope might be reduced due to Roche-lobe overflow during a flash. Sarna et al. (2001) used binary evolution calculations to pursue this idea further. On the other hand, the hydrogen envelope may also be reduced by nuclear burning during the flashes. Indeed, Serenelli et al. (2001) and Rohrmann et al. (2002) find that for masses above $\sim 0.18 M_{\odot}$, their models experience one or more thermonuclear flashes, leading to thinner hydrogen envelopes that cannot sustain nuclear fusion and hence to white dwarfs that cool much faster.

The models are best compared with data. In Table 6, we list the three millisecond pulsars with white dwarf companions for which the mass and temperature are known, and the cooling age can be determined. The first system, PSR J1012+5307, has a pulsar with a characteristic age of $8.9 \mathrm{Gyr}$, and a white dwarf with a temperature of $\sim 8600 \mathrm{~K}$. The other two systems, PSR B1855+09 and PSR J0437-4715, both have ages of $5 \mathrm{Gyr}$ and white dwarf companions with $T_{\text {eff }} \sim 4800 \mathrm{~K}$.

For the cooling ages of the white dwarf companions of these systems to equal to the characteristic pulsar ages, one can readily see that there must a difference in the cooling properties of these white dwarfs. The systems PSR B1855+09 and PSR J0437-4715 are very similar to each other, but rather different from PSR J1012+5307, the latter being older, but, paradoxically having a hotter white dwarf companion. Given the cooling models and the masses of these white dwarfs, it seems likely that this discrepancy is due to a difference in thickness of the hydrogen layer, those of PSR B1855+09 and PSR J0437-4715 being thin and that of PSR J1012+5307 being thick.

Here we report on optical observations of the white dwarf companion of PSR J0218+4232, which, as we will explain below, is expected to have a mass that is between the masses of PSR J1012+5307 and PSR J0437-4715, and thus, potentially, may help us gain insight in the cooling of white dwarfs. In Sect. 2, we outline our current knowledge of the PSR J0218+4232 system. We describe our Keck observations in Sect. 3, from which, in Sect. 4, we determine the white dwarf temperature and surface gravity, together with radial-velocity measurements. The mass of the white dwarf and the age and distance of the system are described in Sect. 5 and we discuss and conclude in Sect. 6.

\section{PSR J0218+4232}

PSR J0218+4232, a 2.3 ms pulsar, was discovered by Navarro et al. (1995). From radio timing observations, they found that it resides in a 2 day circular $\left(e<2 \times 10^{-5}\right)$ orbit around a low mass object, with, assuming a pulsar mass of $1.4 M_{\odot}$, a minimum mass of $0.17 M_{\odot}$. Using the statistical argument that the probability of finding the orbit with an inclination $i$ less than $i_{0}$ is given by $P\left(i<i_{0}\right)=1-\cos i_{0}$, we can conclude that, with $90 \%$ confidence, the mass of the companion is $0.17 \leq$ $M_{\mathrm{WD}} / M_{\odot} \leq 0.44$, the range in which one would expect the object to be a white dwarf with a helium-core.

From considerations of the evolution of binaries containing millisecond pulsars with helium-core white dwarf companions, one expects a relation between the mass of a heliumcore white dwarf companion to a millisecond pulsar and the orbital period (see Joss et al. 1987). This relation has been modelled by Rappaport et al. (1995), Tauris \& Savonije (1999) and others. For the orbital periods of PSR B1855+09 and PSR J0437-4715 (Table 6), the relation by Tauris \& Savonije (1999) predicts companion masses to within the observed errors. Though this relation is not expected to be accurate for orbital periods less than 2 days, the predicted white dwarf mass of PSR J1012+5307, having an orbital period of 0.605 days, is within $1.5 \sigma$ of the observed result. Since the orbital period of PSR J0218+4232 is in between that of PSR J1012+5307 and that of PSR J0437-4715 (5.471 days) it is expected that PSR J0218+4232 has a mass in between their masses. In fact, Tauris and Savonije (1999) predict a mass of $0.216 M_{\odot}$. This is near the mass of $\sim 0.18 M_{\odot}$, where Althaus et al. (2001) predict the transition between thick and thin hydrogen envelopes.

From radio observations, a dispersion measure of $61.25 \mathrm{pc} \mathrm{cm}^{-3}$ was found. In the direction of the pulsar, the Taylor \& Cordes (1993) model for the distribution of interstellar free electrons predicts a minimum distance of $5.7 \mathrm{kpc}$ for this dispersion measure. The quoted uncertainty is about $25 \%$, but may be considerable larger in this direction as there are few pulsars with which the model could be calibrated. Indeed, a new model, presented by Cordes \& Lazio (2002), predicts a distance of $2.7 \mathrm{kpc}$.

The characteristic age of the pulsar is 0.46 Gyr (Navarro et al. 1995). Recent radio observations of PSR J0218+4232 with WSRT/PuMa (B. Stappers, priv. comm.) have reveiled a proper motion, of $\mu=3.97 \pm 1.73 \mathrm{mas} / \mathrm{yr}$. Even for the largest distances we will need to consider, this has negligible effect on the characteristic age, and hence we ignore it below.

\section{Observations}

The location of the PSR J0218+4232 system was observed with the 10-m Keck I telescope at Hawaii, using the lowresolution imaging spectrometer (LRIS, Oke et al. 1995). During the night of 1995 November $21 / 22, R$ and $I$-band images were obtained. The following night single deep $B, V$ and $R$ images were taken. In both nights the seeing was mediocre, 1 ". $0-1$ ". 1 , and the conditions were not photometric. Indeed, the images from the first night were of so much inferior quality 
Table 1. Log of the Keck imaging observations.

\begin{tabular}{|c|c|c|c|}
\hline Object $^{a}$ & Time (UT) & $t_{\text {int }}(\mathrm{s})$ & $\sec z$ \\
\hline \multicolumn{2}{|c|}{1995 November 23} & $B, V, R$ & \\
\hline PSR J0218 & $7: 24-7: 56$ & $1200,900,600$ & $1.09-1.12$ \\
\hline \multicolumn{2}{|c|}{1997 January 9} & $B, V, R, I$ & \\
\hline PSR J0218 & $5: 47-5: 56$ & $120,60,60,60$ & 1.09 \\
\hline PG 0231 & $5: 18-5: 33$ & $2 \times(20,10,10,10)$ & $1.03-1.04$ \\
\hline SA 95 & $5: 36-5: 43$ & $10,10,10,10$ & 1.13 \\
\hline SA 95 & $7: 35-7: 42$ & $10,10,10,10$ & 1.08 \\
\hline PG 1047 & $15: 40-15: 53$ & $2 \times(20,10,10,10)$ & $1.19-1.22$ \\
\hline PG 1323 & $15: 56-16: 02$ & $20,10,10,10$ & 1.15 \\
\hline
\end{tabular}

${ }^{a}$ Full names are PSR J0218+4232, PG 0231+051, PG 1047+003 and PG 1323-086.

than those taken the other night that we did not use them any further.

On the night of 1997 January 8/9 the conditions were photometric, and short exposures of the system were taken in $B$, $V, R$ and $I$-band for photometric calibration. The seeing was again mediocre and varied between 1 ". $0-1$ '.2. Four standard fields (Landolt 1992; Stetson 2000) were also observed in the same bands. Table 1 shows the log of the imaging observations.

Spectra of the companion of PSR J0218+4232 were obtained with the same instrument, now at Keck II, on the nights of 1998 December $16 / 17$ and $17 / 18$. The 600 lines $\mathrm{mm}^{-1}$ grating was used, covering the wavelength range 3500-6000 $\AA$ at $1.25 \AA \mathrm{pix}^{-1}$. The width of the slit was $1^{\prime \prime}$, resulting in a resolution of $\sim 6 \AA$. The seeing varied between 0 ". $8-1$ ". 0 on both nights.

On both nights that the pulsar companion was observed, the white dwarfs WD 0030+444 and WD 0518+333 (Reid 1996) were observed by way of radial-velocity standards, and the spectrophotometric standard G 191B2B (Bohlin et al. 1995) was observed for flux calibration. Exposures of a $\mathrm{HgKrXe}$ lamp were taken for wavelength calibration. All spectra were taken at a position angle close to the parallactic one, to minimize loss of light and velocity shifts due to differential refraction. For PSR J0218+4232, the angle was chosen such that there would be another nearby star in the slit. The spectroscopic observations are tabulated in Table 2.

\subsection{Astrometry}

For astrometric calibration, we selected all 85 stars from the USNO-A2.0 Catalog (Monet et al. 1998) that overlapped with a 10 second $R$-band image obtained on November 23, 1995. Of these stars, 57 were not over exposed and appeared stellar and unblended. Their centroids were measured and corrected for instrumental distortion using a bicubic function determined by J. Cohen (1997, priv. comm.). These points were fitted for zeropoint position, scale and position angle against the USNOA2.0 positions. After rejecting 4 outliers, that had residuals larger than 0 ". 6 , the rms residuals were 0 ". 18 and $0 "$.'22 in right
Table 2. Log of the Keck spectroscopic observations.

\begin{tabular}{lccr}
\hline \hline Object & Time $(\mathrm{UT})$ & $t_{\text {int }}(\mathrm{s})$ & $\sec z$ \\
\hline 1998 December 17 & & & \\
WD 0030+444 & $5: 47$ & 180 & 1.11 \\
PSR J0218+4232 & $6: 05-9: 16$ & $4 \times 2700$ & $1.08-1.26$ \\
WD 0518+333 & $9: 24$ & 180 & 1.04 \\
G 191B2B & $9: 36$ & 30 & 1.19 \\
1998 December 18 & & & \\
WD 0030+444 & $5: 00$ & 180 & 1.10 \\
PSR J0218+4232 & $5: 11-8: 24$ & $4 \times 2700$ & $1.08-1.17$ \\
WD 0518+333 & $8: 34$ & 180 & 1.08 \\
G 191B2B & $8: 48$ & 30 & 1.21 \\
\hline
\end{tabular}

ascension and declination respectively, consistent with the expected errors for USNO-A2.0 positions.

This solution was transferred to the $10 \mathrm{~min} R$-band (and the 20 min $B$-band) image, using 184 (209) stars that appeared on both images and were stellar, unsaturated and not blended. Again the zero-point, scale and position angle were fitted and the final residuals were $<0$ ". $03(<0$ ”.06) in both directions. Thus, our positions should be on the USNO-A2.0 system to within 0 ". 03. The USNO-A2.0 system is on the International Celestial Reference System (ICRS) to within about 0". 2 .

This latter uncertainty is much larger than the measurement error for our optical position, and also greatly exceeds the precision with which the very precise timing position (Navarro et al. 1995; B. Stappers 2003, priv. comm.) can be tied to the ICRS. Within this uncertainty, our images show a single object, "X" hereafter, at a position coincident with the timing position (see Fig. 1). This object is also present on the 1997 images.

\subsection{Photometry}

We used the DAOPHOT II package (Stetson 1987) for the photometry. First, all images were bias-subtracted and flat-fielded using twilight flats. Next, we determined a point spread function (PSF) for each object image from a selection of relatively isolated and single stars. We determined instrumental magnitudes by fitting this PSF to all stars. These fitting results were then used to remove all stars, except those used in determining the PSF. Using aperture photometry we determined the magnitudes of these PSF stars and calculated offsets between the PSF magnitude and the aperture magnitude.

Instrumental magnitudes of the standards stars were determined using aperture magnitudes. This was necessary since the images of the standard fields were deliberately taken out of focus to ensure that the stars would not saturate the detector. Using values of Stetson (2000) we determined magnitude offsets, colour and extinction coefficients to calibrate the 1997 images. The rms residuals after the calibration are smaller than $0.02 \mathrm{mag}$.

Finally, magnitude offsets between the 1995 and 1997 images were determined to transfer the photometric calibration to the 1995 images. We find that for $B, V$ and $R$ respectively $90 \%$, 


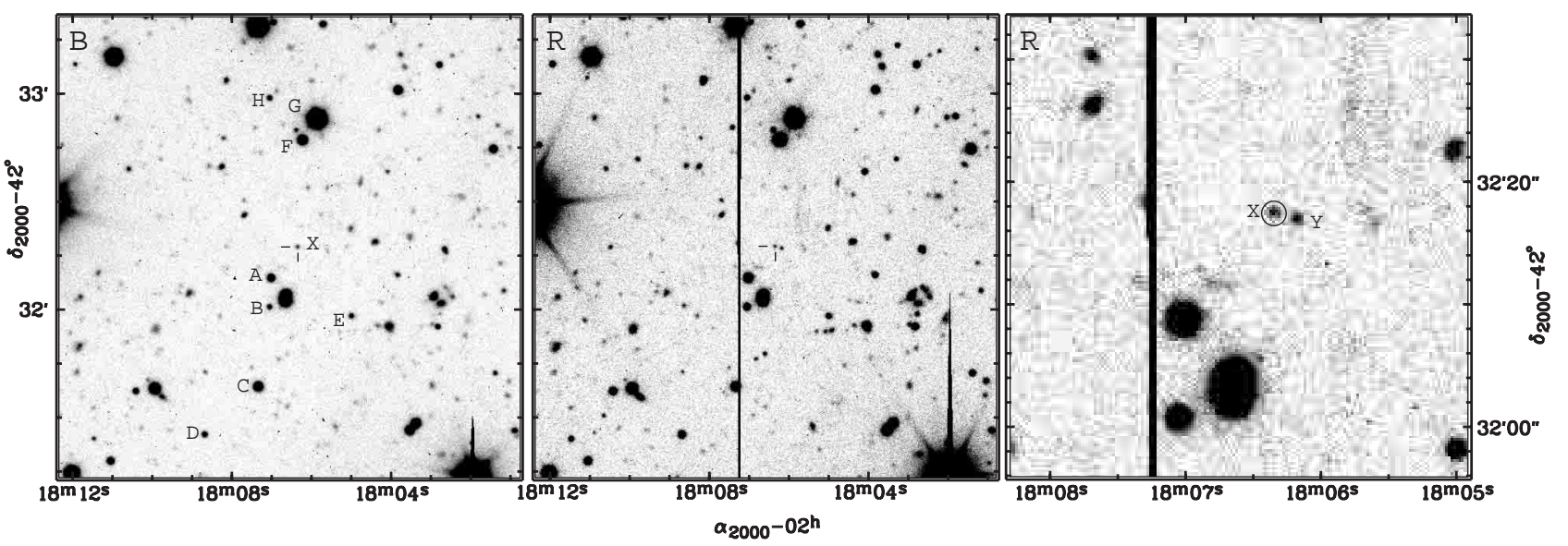

Fig. 1. Optical images of the location of PSR J0218+4232 and its companion. The left-hand panel shows the $1200 \mathrm{~s} B$ image, the middle panel the $600 \mathrm{~s} R$-band image, and the right-hand panel a zoom-in on the $R$-band image. In the latter, the circle indicates the timing position of Navarro et al. (1995). For clarity, it has been drawn with a radius of 1", twice the $95 \%$ confidence error radius. We find one object within the circle and name it object X. Other stars in this field are also named and magnitudes of these stars are given in Table 3. Object Y, clearly visible in $R$ but very faint in $B$, is only $2^{\prime \prime}$ west of the pulsar location.

Table 3. Astrometry and photometry of the companion of PSR J0218+4232 and stars in the field. The nomenclature of the stars is according to Fig. 1. The uncertainties for star X, the pulsar companion, are as listed. For the other stars, the positions are good to within 0 ".01 except for those marked with a colon, which are good to within 0 ".02. For the photometry of the other stars, the presence of no, a single or a double colon indicates uncertainties of $\sigma \leq 0.025$, $0.025<\sigma \leq 0.075$ and $\sigma>0.075 \mathrm{mag}$, respectively. These errors are instrumental, i.e., they do not include the zero-point uncertainty of the astrometric tie $\left(00^{\prime \prime} 2\right.$ in each coordinate) or of 0.02 mag in the photometry. The $B, V$ and $R$ magnitudes are determined from the 1995 images while the $I$-band magnitudes are found from the $60 \mathrm{~s}$ image taken in 1997, hence the larger $I$-band error for object X.

\begin{tabular}{|c|c|c|c|c|c|c|}
\hline \multirow[b]{2}{*}{ ID } & \multicolumn{3}{|c|}{ 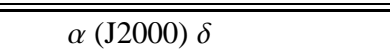 } & \multirow[b]{2}{*}{$V$} & \multirow[b]{2}{*}{$R$} & \multirow[b]{2}{*}{$I$} \\
\hline & $2^{\mathrm{h}} 18^{\mathrm{m}}$ & $+42^{\circ}$ & $B$ & & & \\
\hline \multirow[t]{2}{*}{$\mathrm{X}$} & $06^{\mathrm{s}} 364$ & $32^{\prime} 17^{\prime \prime} 40$ & 24.40 & 24.15 & 23.86 & 23.76 \\
\hline & \pm 0 ”.03 & $\pm 0 "$ "03 & \pm 0.04 & \pm 0.04 & \pm 0.06 & \pm 0.25 \\
\hline A & $07^{\mathrm{s}} .029$ & $32^{\prime} 08^{\prime \prime} .81$ & 21.79 & 20.35 & 19.43 & 18.65 \\
\hline B & $07^{\mathrm{s}} .066$ & $32^{\prime} 00^{\prime \prime} .79$ & 23.43 & 21.88 & 20.86 & 19.77 \\
\hline $\mathrm{C}$ & $07^{\mathrm{s}} 353$ & $31^{\prime} 38^{\prime \prime} 67$ & 20.73 & 20.00 & 19.55 & 19.18 \\
\hline $\mathrm{D}$ & $08^{\text {s.700 }}$ & $31^{\prime} 25^{\prime \prime} 35$ & 23.09 & 21.60 & 20.62 & 19.67 \\
\hline $\mathrm{E}$ & $05^{\mathrm{s}} .015:$ & $31^{\prime} 58^{\prime \prime} .27$ & 23.46 & 22.55 & 21.98 & 21.44: \\
\hline $\mathrm{F}$ & $06^{\mathrm{s}} .238$ & $32^{\prime} 47^{\prime \prime} 09$ & 20.64 & 19.02 & 17.88 & 16.58 \\
\hline $\mathrm{G}$ & $05^{\mathrm{s}} .868$ & $32^{\prime} 52^{\prime \prime} 90$ & 17.23 & 16.81 & 16.42: & 16.15 \\
\hline $\mathrm{H}$ & $07^{\mathrm{s}} .058$ & $32^{\prime} 58^{\prime \prime} .75$ & 23.30 & 22.69 & 22.18 & 21.41: \\
\hline Y & $06^{\mathrm{s}} .194:$ & $32^{\prime} 17^{\prime \prime} 00:$ & $26.37::$ & $24.78::$ & 23.67: & 22.19: \\
\hline
\end{tabular}

$76 \%$ and $85 \%$ of the flux actually reached the detector, consistent with the presence of thin cirrus during the 1995 observations. The magnitudes of the pulsar companion and other stars in the vicinity are tabulated in Table 3 . We estimate the final uncertainty in the zero points at about $0.02 \mathrm{mag}$.

\subsection{Spectroscopy}

All frames were bias-corrected and sky-subtracted using standard procedures. The spectra were extracted using an optimal extraction method similar to that presented by Horne (1986). Each extracted spectrum was wavelength calibrated using the arc lamp exposures, with a correction as detailed below. Then, each individual spectrum was flux calibrated using the observation of $G$ 191B2B of that night.

The spectra of PSR J0218+4232 were extracted using the spatial profile determined from the brighter star that we had made sure was on the slit. After extraction, flux and wavelength calibration, all four spectra of each night were added and averaged. The average of the two averaged spectra is shown in Fig. 2. The Balmer lines $\mathrm{H} \beta, \mathrm{H} \gamma$ and $\mathrm{H} \delta$ are clearly visible, indicating a hydrogen atmosphere, in line with the object being a helium-core white dwarf.

As we hoped to determine a radial-velocity orbit, we took particular pains in trying to verify our wavelength calibration and velocity scale. In the process, we encountered a number of problems, which we detail below. As will become clear in the remainder of this Section, we have not solved the problems, and hence there is an additional uncertainty in the velocities we derive. While in all likelihood this is smaller than the rather large measurement errors for PSR J0218+4232, we still will treat our velocities with caution. We stress, though, that the problems we encountered should have no effect on the main results of this paper, which rely on the temperature determination from the spectra.

As a first correction to the wavelength calibration, we measured the wavelength difference between the observed and known wavelength of the O I $\lambda 5577$ night-sky emission line. We found that these differences had a rms of $0.7 \AA$. We corrected all spectra appropriately.

Next, we determined radial velocities of the two radialvelocity standards for each night by fitting a 9 parameter profile to each line, $\mathrm{H} \beta$ up to $\mathrm{H} 9$, separately. This profile consisted of a parabolic term, added to the sum of a Lorentzian and a 

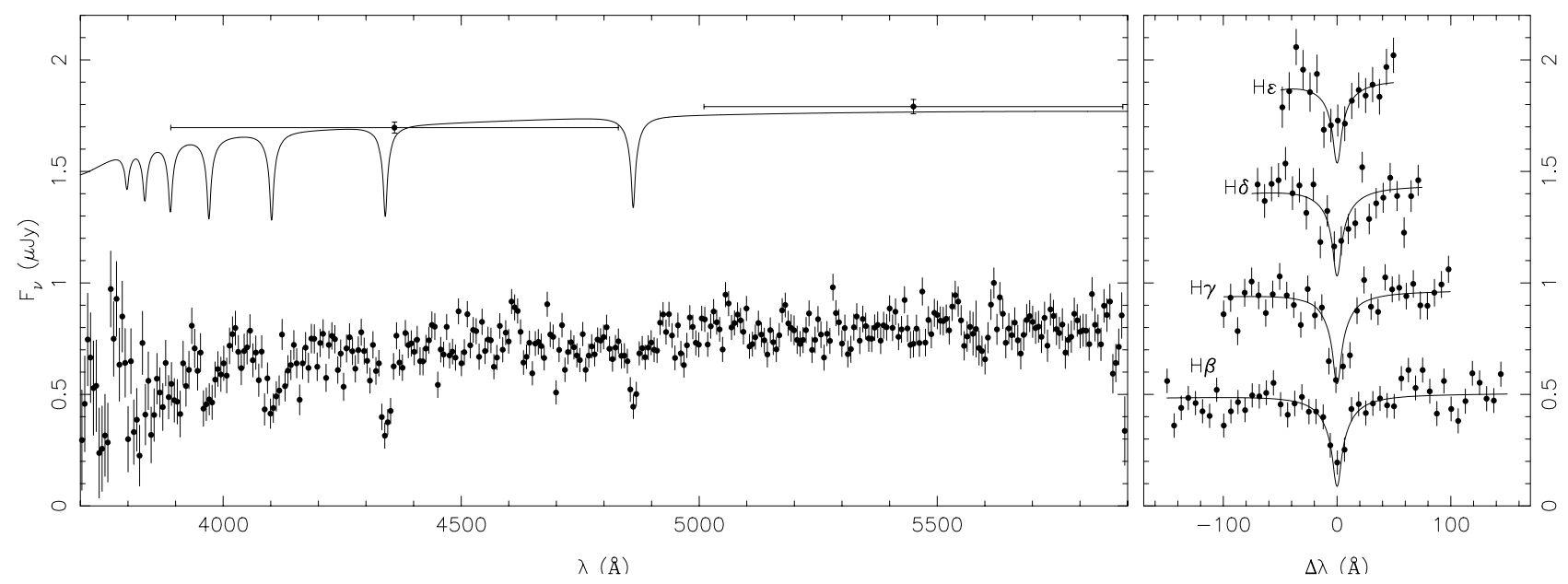

Fig. 2. The spectrum of the companion of PSR J0218+4232. Shown in the left-hand panel is the average of the observed spectra when shifted to zero-velocity (bottom curve) and the convolved modelled spectrum with $T_{\text {eff }}=8000 \mathrm{~K}$ and $\log g=7.0 \mathrm{~cm} \mathrm{~s}^{-2}$ (top curve), shifted $1 \mu \mathrm{Jy}$ upwards. Superposed to the latter are the observed $B$ and $V$ broadband fluxes (also offset by $1 \mu \mathrm{Jy}$ ). The right-hand panel shows the $\mathrm{H} \beta$ to $\mathrm{H} \epsilon$ lines from the observed spectrum with the convolved model superposed.

Gaussian, both having the same centre, but different widths and amplitudes. This profile fitted the lines extremely well, but unfortunately we found that the resulting velocities for the different lines were inconsistent with each other.

After correcting for the barycentric motion of the Earth, the velocity determined from the $\mathrm{H} \beta$ line in both spectra of WD 0518+333 was consistent with the results from Reid (1996), while the other lines gave higher velocities. In general, the $\mathrm{H} \gamma, \mathrm{H} \delta$ and $\mathrm{H} \epsilon$ lines gave velocities that were approximately 56,78 and $80 \mathrm{~km} \mathrm{~s}^{-1}$ too red. The velocities determined from $\mathrm{H} 8$ and $\mathrm{H} 9$ had too large an error to be meaningful.

For the spectrum of WD 0030+444 taken on the first night, we found a similar result, with the $\mathrm{H} \beta$ velocity in agreement with the known value and the velocities of other lines following the pattern described above. The results for the second night, however, differed from those of the first night. First, the $\mathrm{H} \beta$ velocity was slow by $25 \mathrm{~km} \mathrm{~s}^{-1}$, and second, the other velocities followed a different pattern.

To verify our methods, we used the flux calibration standard $\mathrm{G}$ 191B2B, which is a hot sub-dwarf and which also has the $\mathrm{H} \beta$ to $\mathrm{H} \epsilon$ lines in its spectra, though they are much less wide than those of WD $0518+333$ and WD $0030+444$. Its radial velocity has not been determined as accurately, but we can still use it for relative calibration. We determined velocities with the same method as described above, and found that these followed the same pattern as the observations of WD $0518+333$ and the first observation of WD $0030+444$.

On closer inspection, we found that the observation of WD $0030+444$ on the second night differed in flux from the observation on the first night, the second spectrum having $40 \%$ fewer counts. Using the filter curve from Bessell (1990), we calculated synthetic $B$-band magnitudes for both spectra. We found that the first spectrum gave a magnitude consistent with the literature value, while the second spectrum obviously did not. Since there was no difference in airmass and conditions, we believe that WD $0030+444$ was incorrectly placed on the slit, thus causing the inconsistent velocities and the flux
Table 4. Radial velocity measurements of the radial velocity standards WD 0030+444 and WD 0518+333 and the spectrophotometric standard G 191B2B.

\begin{tabular}{cccr}
\hline \hline Date (UT) & \multicolumn{3}{c}{$v\left(\mathrm{~km} \mathrm{~s}^{-1}\right)$} \\
& WD 0030+444 & WD 0518+333 & G191B2B \\
\hline 1998 Dec. 17 & $78.8 \pm 3.2$ & $34.7 \pm 2.3$ & $22.3 \pm 4.0$ \\
1998 Dec. 18 & $35.1 \pm 4.4^{a}$ & $34.7 \pm 2.6$ & $9.9 \pm 4.3$ \\
\hline
\end{tabular}

${ }^{a}$ We believe this radial velocity measurement is in error due to misplacement of this object onto the slit.

difference. Therefore, we decided not use the observation of WD $0030+444$ on the second night any further.

We tried to find a reason for the trend in velocity found in the different Balmer lines, but could not find any. Therefore, we simply used the velocities found from $\mathrm{H} \beta, \mathrm{H} \gamma, \mathrm{H} \delta$ and $\mathrm{H} \epsilon$ (H8 and H9 had too large an error) to calculated a new empirical wavelength scale, depending linearly on the wavelength calibration found from the arc lamp measurements. This correction was applied to all spectra, after applying the shift for the wavelength difference found from the night-sky line.

With both corrections in place, the resulting velocities (see Table 4) have root-mean-square errors of approximately $14 \mathrm{~km} \mathrm{~s}^{-1}$. We take this as an estimate of the additional uncertainty in any velocity measurement, but stress that since we do not know the cause of the problem with the wavelength calibration, we cannot be certain it applies to all observations. As noted, however, it should not influence our main results, which rely on the temperature determination.

\section{Temperature, gravity and radial velocities}

We use the photometry and the spectra of the companion of PSR J0218+4232 to constrain its effective temperature and surface gravity by comparing them with model atmospheres. Furthermore, we determine radial velocities of the two spectra 
and use these to estimate the mass ratio between the pulsar and the white dwarf and the systemic velocity of the system.

We take into account the reddening in the direction of the pulsar. Schlegel et al (1998) give $E_{B-V}=0.07 \pm 0.01$ and we correct our magnitudes accordingly. This reddening value was also used to correct the observed spectra using relations given by O'Donnell (1994).

The two separate average spectra of the companion, taken on the two subsequent days, were compared with model spectra for pure hydrogen atmospheres, kindly provided by P. Bergeron (2002, priv. comm.), to infer the effective temperature and the surface gravity. The grid of models used spanned $T_{\text {eff }}=7000$ (500) $9500 \mathrm{~K}$ and $\log g=6.5(0.5) 8.0 \mathrm{~cm} \mathrm{~s}^{-2}$, where the number in parentheses is the step between the models. We convolved each model with a cut-off Gaussian to mimic the effects of seeing and the slit. This convolved model was then fitted to each observed spectrum, using data-points around $\mathrm{H} \beta$ up to $\mathrm{H} \epsilon$, leaving the radial velocity $v$, flux normalisation $A$ and slope $\alpha$ to be fitted by minimising the $\chi^{2}$ merit function, defined by

$\chi^{2}=\sum_{i}\left(\frac{f_{i}-f\left[(1+v / c) \lambda_{i}\right] \times A\left(\lambda_{i} / 4340 \AA\right)^{\alpha}}{\sigma_{i}}\right)^{2}$.

Here $f_{i}$ and $\sigma_{i}$ are the observed flux and error at wavelength $\lambda_{i}$, and $f[\lambda]$ is the model flux at wavelength $\lambda$. We introduce the slope $\alpha$ to account for possible errors in the (relative) flux calibration.

For each of the two observed spectra a surface of $\chi^{2}$ values in the $T_{\text {eff }}, \log g$ plane, spanned by the modelled spectra, was created. For each model spectrum the $\chi^{2}$ value of the first spectrum was added to that of the second to create a third surface. In Fig. 3, we show contours and the location of the minimum in this latter surface; the surfaces found from the individual spectra are similar in shape. At the minimum, we find $\chi_{\min }^{2}=778.8$. As we have 606 degrees of freedom, this indicates that the fit is satisfactory, but that likely we underestimated our errors slightly. The errors in temperature and surface gravity were rescaled accordingly. The best-fit power-law slopes for the two spectra are small, $-0.11 \pm 0.16$ and $0.21 \pm 0.19$ for the first and second night, respectively, indicating that our relative flux calibration was reasonably good. Indeed, also the absolute calibration is fair: the $B$-band magnitude, using the filter curve by Bessell (1990), from our observed spectra is 24.43, within the error of the photometric measurement.

From Fig. 3, one sees that our spectra constrain the temperature fairly well $\left(T_{\text {eff }}=8060 \pm 150 \mathrm{~K}\right)$, but provide only a very weak constraint on the gravity $\left(\log g=6.9 \pm 0.7 \mathrm{~cm} \mathrm{~s}^{-2}\right)$. This is because the gravity mostly affects the higher Balmer lines, at wavelengths shortwards of $4000 \AA$, where our spectra are very noisy.

The photometric colours of the companion can also be used to infer a temperature. From atmosphere models by Bergeron et al. (1995), we find a temperature of $T_{\text {eff }}=8560 \pm 700 \mathrm{~K}$ for a pure hydrogen atmosphere with $\log g=8$. Serenelli et al. (2001) and Rohrmann et al. (2002) calculated evolutionary tracks of the formation and cooling of helium white dwarfs. They also give colours based on model atmospheres of Rohrmann (2001). From these we infer a temperature of

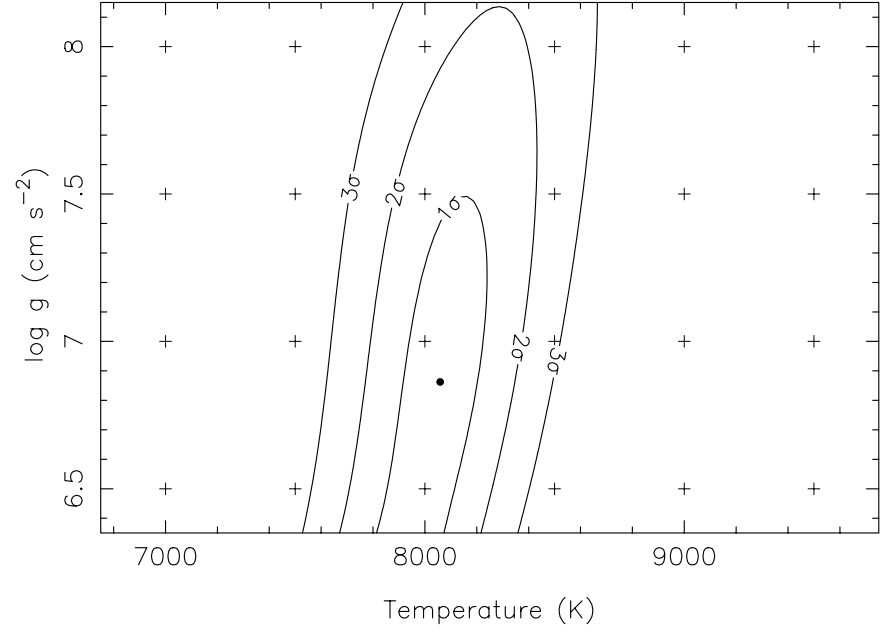

Fig. 3. Confidence intervals on effective temperature $T_{\text {eff }}$ and surface gravity $\log g$ as inferred from our spectra. The plus signs indicate the parameter combinations of the models in our grid. At each point, the best-fit $\chi^{2}$ was determined, leaving velocity, flux amplitude, and spectral slope free to vary. The results were interpolated to create a $\chi^{2}$ surface, which has a minimum at the position shown with a filled circle, at $T_{\text {eff }}=8060 \pm 150 \mathrm{~K}$ and $\log g=6.9 \pm 0.7 \mathrm{~cm} \mathrm{~s}^{-2}$. The three contours, at $\chi_{\min }^{2}$ plus 1,4 and 9 , delineate the $1 \sigma, 2 \sigma$ and $3 \sigma$ error regions.

Table 5. Radial velocity measurements of the companion of PSR J0218+4232. The uncertainties listed are measurement errors, and do not take into account the possible systematic errors associated with the problems in the wavelength calibration (Sect. 3.3).

\begin{tabular}{ccccr}
\hline \hline \multicolumn{2}{c}{ Date \& time (UT) } & MJD bar $_{\text {br }}$ & $\phi_{\text {orb }}^{a}$ & $v\left(\mathrm{~km} \mathrm{~s}^{-1}\right)$ \\
\hline 1998 Dec. 17 & $07: 45$ & 51164.3281 & 0.544 & $-211 \pm 64$ \\
1998 Dec. 18 & $06: 52$ & 51165.2891 & 0.018 & $101 \pm 78$ \\
\hline
\end{tabular}

${ }^{a}$ Using the ephemeris of Navarro et al. (1995):

$T_{0}=$ MJD $_{\text {bar }} 49150.6086, P_{b}=2.0288461$ days.

$T_{\text {eff }}=8400 \pm 500 \mathrm{~K}$ over a range of surface gravities ( $\log g=$ 6.0-7.5). Both these temperatures are consistent with what we found from our spectra.

The radial velocities that we found from fitting the spectra to the models by Bergeron are given in Table 5 . These velocities are corrected for the barycentric motion of the earth and are for the best fit of $T_{\text {eff }}$ and $\log g$. The error estimates include the extra uncertainty in each velocity due to the uncertainties in the temperature, gravity, normalisation, and slope.

The two radial velocities were fitted to a circular orbit using the ephemeris of Navarro et al. (1995). We find a systemic velocity of $\gamma=-57 \pm 52 \mathrm{~km} \mathrm{~s}^{-1}$ and a radial-velocity amplitude of $K_{\mathrm{WD}}=159 \pm 50 \mathrm{~km} \mathrm{~s}^{-1}$. Naturally, this is a perfect fit, because we use two data points to determine two unknowns, i.e., we have no degrees of freedom and thus no information about the correctness of the fit. This makes the systemic velocity and the velocity amplitude very dependent on any error in the determinations of the radial velocities. We note, though, that possible systematic errors in the radial-velocity determinations have less effect on the velocity amplitude than on the systemic velocity, 
because, to first order, such errors should cancel out when determining the velocity amplitude, while being compounded in the systemic velocity.

From the radial velocity amplitude of the white dwarf and the pulsar, $21.3243 \pm 0.0001 \mathrm{~km} \mathrm{~s}^{-1}$, (Navarro et al. 1995), it follows that $q \equiv M_{\mathrm{PSR}} / M_{\mathrm{WD}}=7.5 \pm 2.4$.

\section{Mass, age and distance}

With our effective temperature and estimate of the surface gravity, we can use evolutionary models to estimate the mass and age of the white dwarf as well as the distance of the system.

\subsection{White dwarf mass}

The weak constraint that our spectroscopic observations place on the surface gravity translate into a similarly weak constraint on the mass. Using the models of Rohrmann et al. (2002), which span the range of $0.148-0.406 M_{\odot}$, we find a white dwarf mass of $M_{\mathrm{WD}}=0.21_{-0.04}^{+0.17} M_{\odot}$.

Similar values are found using the white dwarf evolution models of Driebe et al. (1998). We stress, though, the uncertainty: the upper $2 \sigma$ error on the surface gravity is $\log g=8$ (see Fig. 3), which corresponds to a mass of about $0.6 M_{\odot}$ which is above the range expected for a helium-core white dwarf.

We can also use the mass ratio, determined above, to estimate the mass of the white dwarf. If we assume a pulsar mass of $1.48 \pm 0.08 M_{\odot}$, the weighed average of pulsar masses from Table 6 (excluding that of PSR J0218+4232), this results in a white dwarf mass of $M_{\mathrm{WD}}=0.20 \pm 0.06 M_{\odot}$, in line with what we determine from the surface gravity. The pulsar mass, as predicted by our observed values, is $M_{\mathrm{PSR}}=1.6_{-0.7}^{+2.2} M_{\odot}$.

\subsection{Distance and age}

To compare the white dwarf cooling age with the characteristic age of the pulsar we need to know the white dwarf's mass and temperature, as well as the distance to the system. The latter can be found from comparison with the white dwarf's absolute magnitude and our photometry, but for this we, again, need to know the mass of the white dwarf.

Since our constraint on the white dwarf mass is weak, we invert the procedure. For a given white dwarf model and a given mass, we compare our temperature with the predictions from the model to infer a cooling age and use the luminosity or the absolute visual magnitude to find a distance.

By comparison of our temperature with the cooling models of Hansen \& Phinney (1998a) and the evolutionary models by Driebe et al. (1998) and Rohrmann et al. (2002) we estimate the cooling age and the distance of the white dwarf as a function of its mass: we show the result in Fig. 4. Each point represents a model with a certain mass. The errors in the distance and the age are caused by the uncertainty in the effective temperature of the white dwarf and, to a lesser extent, the uncertainty in the observed magnitudes. The errors are depicted by diagonal bars instead of horizontal and vertical ones, because the uncertainty in the distance is correlated with the uncertainty in the age, as

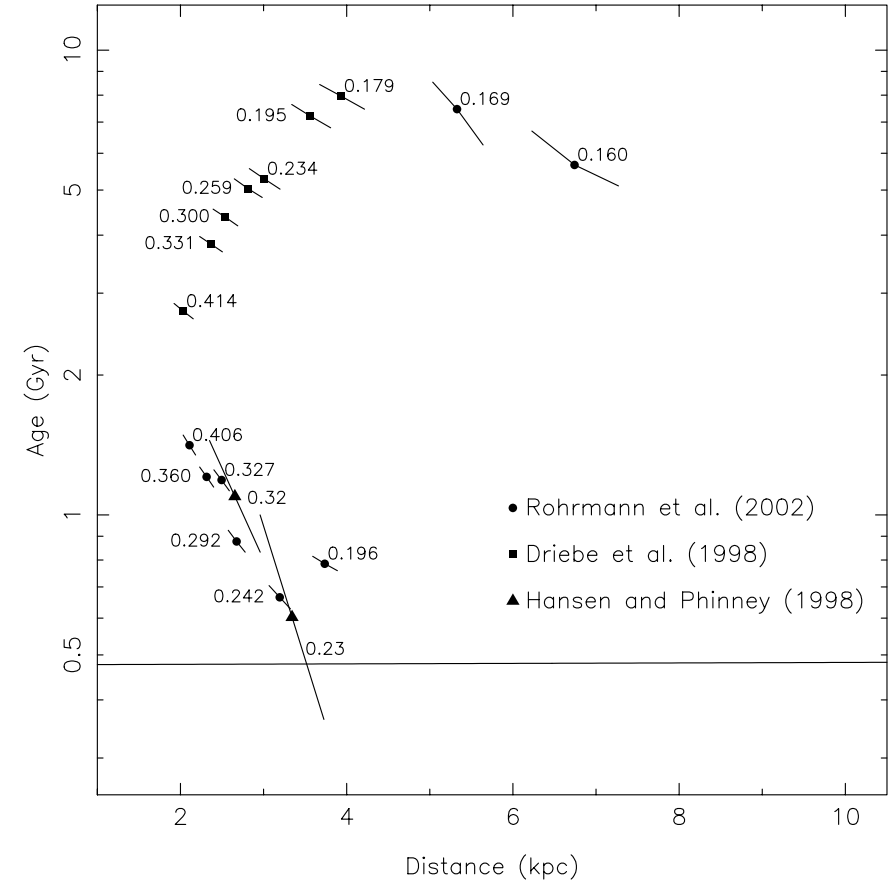

Fig. 4. The white dwarf cooling age as a function of distance. For each model, we determine the distance and cooling age for our observed temperature and magnitude. Since this, through the model, creates a correlation between the distance and cooling age, we use diagonal error bars. The two Hansen and Phinney models have larger errors, because we had to use our more uncertain $I$-band magnitude. The dots are the Rohrmann models, the squares the Driebe models and the triangles the Hansen and Phinney models. The solid curve denotes the characteristic pulsar age.

both depend on the white dwarf temperature. The horizontal line depicts the characteristic age of the pulsar.

Figure 4 shows that, for this temperature and observed magnitude, there is a clear distinction between the models predicting thin hydrogen envelopes and those predicting thick envelopes. All models by Driebe et al. (1998) and the two lowest mass models by Rohrmann et al. (2002) have thick hydrogen envelopes and predict white dwarf cooling ages that are much older than the characteristic age of the pulsar. The Hansen \& Phinney (1998a) models and the Rohrmann et al. (2002) models with masses above $0.18 M_{\odot}$ have thin hydrogen envelopes and hence predict younger white dwarfs at the temperature found for the companion of PSR J0218+4232. If the age of the pulsar is indeed roughly equal to the characteristic age, then the white dwarf companion must have a thin hydrogen envelope.

The second thing to note is the close agreement with which the different groups predict the distance for a given mass. Since the luminosity we observe depends on the temperature, distance and radius of the white dwarf, similar distances at a given temperature indicate similar radii. Apparently, the radius is set by the degenerate core, the thickness of the hydrogen envelope having little influence at this temperature.

Returning to the comparison between the characteristic age of the pulsar and the cooling age of the white dwarf we can conclude that helium-core white dwarfs with thin hydrogen envelopes predict ages that are most similar, but a little older, to 
Table 6. White dwarf companions to millisecond pulsars. The mass and temperature of the white dwarf in these systems is known accurately. Listed here are the orbital period, the mass of the white dwarf and of the pulsar, the temperature of the white dwarf and the characteristic age of the pulsar.

\begin{tabular}{lcccccc}
\hline \hline Pulsar name & $P_{\mathrm{b}}(\mathrm{d})$ & $M_{\mathrm{WD}}\left(M_{\odot}\right)$ & $M_{\mathrm{PSR}}\left(M_{\odot}\right)$ & $T_{\text {eff }}(\mathrm{K})$ & $\tau_{\text {char }}(\mathrm{Gyr})$ & References \\
\hline PSR J1012+5307 & 0.605 & $0.16 \pm 0.02$ & $1.52 \pm 0.21^{a} / 1.64 \pm 0.22$ & $8550 \pm 25 / 8670 \pm 300$ & $8.9 \pm 1.9$ & $1^{b}, 2^{c d}, 3^{c d}$ \\
PSR J0437-4715 & 5.741 & $0.236 \pm 0.017$ & $1.58 \pm 0.18$ & $\sim 4750$ & $4.9 \pm 0.1$ & $4^{b c}, 5^{d}$ \\
PSR B1855+09 & 12.327 & $0.248 \pm 0.011$ & $1.57 \pm 0.11$ & $4800 \pm 800$ & $4.9 \pm 0.2$ & $6^{b c}, 7^{c}, 8^{d}$ \\
PSR J0218+4232 & 2.029 & $0.21_{-0.04}^{+0.17}$ & $1.6_{-0.7}^{+2.2}$ & $8060 \pm 150$ & $\sim 0.46$ & $9^{b}, 10^{c d}$ \\
\hline
\end{tabular}

References: (1) Lange et al. (2001), (2) van Kerkwijk et al. (1996), (3) Callanan et al. (1998), (4) van Straten et al. (2001), (5) Danziger et al. (1993), (6) Kaspi et al. (1994), (7) Nice et al. (2002), (8) van Kerkwijk et al. (2000), (9) Navarro et al. (1995) and (10) this paper.

${ }^{a}$ Using the white dwarf mass and a mass-ratio of $9.5 \pm 0.5$ (Unpublished work).

${ }^{b}$ Timing observations.

${ }^{c}$ Mass determination.

${ }^{d}$ Temperature determination.

the characteristic age of the pulsar. The best agreement between both ages is found for white dwarfs with masses between 0.19 to $0.30 M_{\odot}$, corresponding to distances of 2.5 to $4 \mathrm{kpc}$ and cooling ages around $0.7 \mathrm{Gyr}$.

\section{Discussion and conclusions}

We have identified the companion of PSR J0218+4232 and presented photometry and spectroscopy. Our spectra show that the companion has a hydrogen atmosphere and from comparison with hydrogen atmosphere models we find that the object has temperature of $T_{\text {eff }}=8060 \pm 150 \mathrm{~K}$ and a surface gravity of $\log g=6.9 \pm 0.7 \mathrm{~cm} \mathrm{~s}^{-1}$.

This temperature and gravity would imply a mass of $M_{\mathrm{WD}}=0.21_{-0.04}^{+0.17} M_{\odot}$, in line with what one would expect for a helium-core white dwarf. Similar white dwarf masses, 0.19 to $0.30 M_{\odot}$, are found when comparing the predicted cooling age of the white dwarf with the characteristic age of the pulsar.

Furthermore, the comparison between the cooling age and the characteristic age shows that this white dwarf companion is likely to have a thin hydrogen envelopes, in agreement with the prediction of Althaus et al. (2001), that the transition between thick and thin hydrogen envelopes is situated around a white dwarf mass of $\sim 0.18 M_{\odot}$.

Thin hydrogen envelopes are also required to match the white dwarf cooling ages with characteristic pulsar ages in the systems PSR B1855+09 and PSR J0437-4715 (Table 6). For PSR J1012+5307, several authors (Alberts et al. 1996; Driebe et al. 1998; Althaus et al. 2001) have argued that a thick hydrogen layer is needed to explain its old age and high temperature. Our mass determination is to weak to put a direct observational constraint on the location of the transition. On the basis of the relation between orbital period and mass, however, we expect that the mass of PSR J0218+4232 is in between those of PSR J1012+5307 and PSR J0437-4715. We can conclude that for whatever mass corresponds to an orbital period of 2 days, thin hydrogen layers are needed.

This empirical constraint may be improved from observations of the white dwarf companions of millisecond pulsars PSR J0034-0534 and PSR J0613-0200, as these systems have orbital periods, 1.6 and $1.1 \mathrm{~d}$ respectively, in between those of PSR J0218+4232 and PSR J1012+5307.

The companion of PSR J0034-0534 has been detected by Lundgren et al. (1996b) who place an upper limit on the temperature of $3800 \mathrm{~K}$. This limit depends on the distance, which was inferred from the dispersion measure. Nevertheless, with a characteristic pulsar age of $5.9 \mathrm{Gyr}$, this white dwarf would likely need a thin hydrogen envelope to have cooled to such a low temperature.

So far, the only observed system for which a thick hydrogen envelope is required, is PSR J1012+5307. We should keep in mind, however, the possibility that this pulsar is actually very young, i.e., that the assumption $P_{0} \ll P$ does not hold. If this were the case then perhaps no white dwarf would need thick hydrogen layers.

This can be verified observationally by measuring the temperature of the white dwarf companion of PSR J0751+1807. This binary has an orbital period of only 6.3 hours, shorter than that of PSR J1012+5307, and hence the white dwarf should have even lower mass. Optical observations by Lundgren et al. (1996a) have not detected an optical counterpart down to $V=$ 23.5, indicating that the white dwarf companion is rather cool ( $T \lesssim 7000 \mathrm{~K}$ for $M<0.2 M_{\odot}$ and $d \simeq 2 \mathrm{kpc}$ ). It would need a thin hydrogen layer to have cooled to such a low temperature within the characteristic age of the pulsar $(8 \mathrm{Gyr})$. Identification of this counterpart and a measurement of its temperature could decide whether it has a thin or a thick hydrogen layer.

Acknowledgements. C.G.B. is indebted to F. Hulleman for help with the photometry. We thank P. Bergeron, A. Serenelli and B. Stappers for very useful discussions about white dwarf atmospheres, white dwarf interiors and radio observations, respectively. The observations reported here were obtained at the W.M. Keck Observatory, which is operated by the California Association for Research in Astronomy. The reduction was done using the Munich Image Data Analysis System, which is developed and maintained by the European Southern Observatory. This research made use of the SIMBAD database. M.H.v.K. acknowledges support of a fellowship of the Royal Netherlands Academy of Arts and Sciences, and S.R.K. of grants from NSF and NASA. 


\section{References}

Alberts, F., Savonije, G. J., van den Heuvel, E. P. J., \& Pols, O. R. 1996, Nature, 380, 676

Althaus, L. G., Serenelli, A. M., \& Benvenuto, O. G. 2001, MNRAS, 324, 617

Bergeron, P., Wesemael, F., \& Beauchamp, A. 1995, PASP, 107, 1047

Bessell, M. S. 1990, PASP, 102, 1181

Bohlin, R. C., Colina, L., \& Finley, D. S. 1995, AJ, 110, 1316

Callanan, P. J., Garnavich, P. M., \& Koester, D. 1998, MNRAS, 298, 207

Camilo, F., Thorsett, S. E., \& Kulkarni, S. R. 1994, ApJ, 421, L15

Cordes, J. M., \& Lazio, T. J. W. 2002, preprint [astro-ph/0207156]

Danziger, I. J., Baade, D., \& Della Valle, M. 1993, A\&A, 276, 382

Driebe, T., Schönberger, D., Blöcker, T., \& Herwig, F. 1998, A\&A, 339,123

Driebe, T., Blöcker, T., Schönberger, D., \& Herwig, F. 1999, A\&A, 350, 89

Hansen, B. M. S., \& Phinney, E. S. 1998a, MNRAS, 294, 557

Horne, K. 1986, PASP, 98, 609

Joss, P. C., Rappaport, S., \& Lewis, W. 1987, ApJ, 319, 180

Kaspi, V. M., Taylor, J. H., \& Ryba, M. F. 1994, ApJ, 428, 713

Landolt, A. U. 1992, AJ, 104, 340

Lange, Ch., Camilo, F., Wex, N., et al. 2001, MNRAS, 326, 274

Lundgren, S. C., Cordes, J. M., Foster, R. S., Wolszczan, A., \& Camilo, F. 1996a, ApJ, 458, L33

Lundgren, S. C., Foster, R. S., \& Camilo, F. 1996b, Pulsars: Problems \& Progress, ed. S. Johnston, M. A. Walker, \& M. Bailes, ASP Conf. Ser., 105, 497

Lyne, A. G., \& Smith, F. G. 1998, Pulsar Astronomy (Cambridge: Cambridge Univ. Press)

Monet, D., et al. 1998, USNO-A2.0 (Washington DC: US Naval Observatory)
Navarro, J., de Bruyn, A. G., Frail, D. A., Kulkarni, S. R., \& Lyne, A. G. 1995, ApJ, 455, L55

Nicastro, L., Lyne, A. G., Lorimer, D. R., et al. 1995, MNRAS, 273, L68

Nice, D. J., Splaver, E. M., \& Stairs, I. H. 2002, preprint [astro-ph/0210637]

O’Donnell, J. E. 1994, ApJ, 422, 158

Oke, J. B., Cohen, J. G., Carr, M., et al. 1995, PASP, 107, 375

Rappaport, S., Podsiadlowski, Ph., Joss, P. C., Di Stefano, R., \& Han, Z. 1995, MNRAS, 273, 731

Reid, I. N. 1996, AJ, 111, 2000

Rohrmann, R. D. 2001, MNRAS, 323, 699

Rohrmann, R. D., Serenelli, A. M., Althaus, L. G., \& Benvenuto, O. G. 2002, MNRAS, 335, 499

Sarna, M. J., Ergma, E., \& Antipova, J. 2000, MNRAS, 316, 84

Sarna, M. J., Ergma, E., \& Gerškevitš, J. 2001, AN, 322, 405

Schlegel, D. J., Finkbeiner, D. P., \& Davis, M. 1998, ApJ, 500, 525

Schönberger, D., Driebe, T., \& Blöcker, T. 2000, A\&A, 356, 929

Serenelli, A. M., Althaus, L. G., Rohrmann, R. D., \& Benvenuto, O. G. 2001, MNRAS, 325, 607

Shklovskii, I. S. 1970, Soviet Astron., 13, 562

Stetson, P. B. 1987, PASP, 99, 191

Stetson, P. B. 2000, PASP, 112, 925

Tauris, T. M., \& Savonije, G. J. 1999, A\&A, 350, 928

Taylor, J. H., \& Cordes, J. M. 1993, ApJ, 411, 674

Thorsett, S. E., \& Chakrabarty, D. 1999, ApJ, 512, 288

van Kerkwijk, M. H., Bergeron, P., \& Kulkarni, S. R. 1996, ApJ, 467, L89

van Kerkwijk, M. H., Bell, J. F., Kaspi, V. M., \& Kulkarni, S. R. 2000, ApJ, 530, L37

van Straten, W., Bailes, M., Britton, M., et al. 2001, Nature, 412, 158

Webbink, R. F. 1975, MNRAS, 171, 555 